\title{
Atomic data for IR and sub-mm wavelengths
}

\author{
Gillian Nave \\ National Institute of Standards and Technology, Gaithersburg, MD, USA \\ email: gnave@nist.gov
}

\section{Introduction}

Atomic spectra in the infrared and sub-mm wavelength regions can be divided into two broad categories: electric dipole-allowed transitions, and forbidden lines due to transitions within the ground term or between low-lying levels of the same parity. Both are of potential importance in the interpretation of astrophysical spectra. Allowed transitions can provide diagnostic information for stellar photospheres, particularly for elements that are not accessible in the visible region. Electric-dipole forbidden lines are important diagnostics of low-density plasmas, such as nebulae and the interstellar medium. In order to interpret astrophysical spectra, accurate atomic data are required. This paper summarizes the techniques for measuring atomic data and lists the most important compilations and databases.

\section{Techniques for measuring IR atomic data}

Forbidden line wavelengths: Forbidden lines from neutral through doubly-ionized species are rarely observed in the laboratory. Although wavelengths of a few lines have been directly measured using laser spectroscopy (e.g. Cooksy, Blake, \& Saykally 1986), or by analysis of astronomical spectra (e.g. Feuchtgruber, Lutz \& Beintema 2001), accurate wavelengths for these lines are usually determined from optimized energy levels. The most common technique is to measure wavelengths of allowed lines in the visible and ultraviolet regions using Fourier transform spectroscopy (FTS) (e.g. Aldenius \& Johansson 2007) and use these to measure the energy levels involved in the forbidden transition. This technique is limited to neutral through doubly-ionized species with resonance lines above about $140 \mathrm{~nm}$. This includes most of the transition-group elements.

Forbidden line oscillator strengths: The majority of the oscillator strengths of forbidden lines in atomic spectra databases are from atomic structure calculations. However, some measurements have been made by combining lifetimes obtained from laser-excitation of ions in a storage ring with branching fractions obtained from astrophysical spectra (e.g. Hartman et al. 2008).

Allowed line wavelengths: Electric dipole-allowed lines in the IR region can originate from low-excitation levels, but most are due to transitions between highly-excited levels. Wavelengths for both can be measured by Fourier transform spectroscopy. Extensive lists of IR data include Ne I (Sansonetti, Blackwell \& Saloman 2004), Ar I (Whaling et al. 1995), Ar II (Whaling et al.), Ti I (Forsberg 1991), Fe I (Nave et al. 1994), Co I (Pickering \& Thorne 1996 ), Co II (Pickering et al. 1998), Ni I (Litzén et al. 1993), and Kr I (Sansonetti \& Greene 2007). Some rare earth elements also have their principal resonance lines in this region (e.g. Ce III, Johansson \& Litzén 1972 ).

Allowed line oscillator strengths: There have been few measurements of atomic oscillator strengths in the IR. Some of the most extensive include Fe I (O'Brian et al. 1991), Ti I (BlackwellWhitehead et al. 2005) and Mn I (Blackwell-Whitehead et al. 2006). The latter two papers are part of a collaborative effort to obtain IR atomic data for cool star analysis by Lund University, Sweden, Imperial College London, UK, and NIST, USA. All have been measured by combining branching fraction measurements using Fourier transform spectroscopy with level lifetimes using laser-induced fluorescence.

IR wavelength calibration: Wavelengths of Th/Ar hollow cathode lamps suitable for calibration of IR spectrographs have been published by Kerber, Nave \& Sansonetti (2008). Measurements of other elements of potential interest for spectrograph calibration are contained in a companion paper (Kerber et al. 2009). 


\section{Databases and compilations}

The last compilation of atomic data dedicated to the IR region was by Outred (1978). Another valuable list of line identifications in the IR region is the key to identification of solar features in the ATMOS spectrum by Geller (1992). Additional databases including IR atomic data include the NIST Atomic Spectra Database, the Vienna Atomic Line Database, the Atomic linelist at Kentucky and Kurucz atomic line database. In addition to atomic data for allowed transitions, these databases also include wavelengths and calculated oscillator strengths for forbidden lines.

\section{References}

Aldenius, M. \& Johansson, S. 2007, A\& A 467, 753.

Atomic linelist at Kentucky http://www.pa.uky.edu/peter/atomic/

Blackwell-Whitehead, R. J., Xu, J. L., Pickering, J. C., Nave, G., \& Lundberg, H. 2005 MNRAS $361,1281$.

Blackwell-Whitehead, R. J., Lundberg, H, Nave, G., Pickering, J. C., Jones, H. R. A, Lyubchik, Y., Pavlenko, Y. V., \& Viti, S. 2006 MNRAS 373, 1603.

Cooksy, A. L., Blake, G. A., \& Saykally, R. J. 1986, ApJ 305, L89.

Forsberg, P 1991, Phys. Scr 44, 446.

Feuctgruber, H. Lutz, D., \& Beintema, D. A 2001 ApJS 136, 221.

Geller, M., 1992 NASA ref. publ. 1224, vol III.

Hartman, H., Gurell, J., Lundin, P., Schef, P., Hibbert, A., Lundberg, H., Mannervik, S., Norlin, L.-O., \& Royen, P. 2008, A\&A 480, 571.

Johansson, S. \& Litzén, U. 1972, Phys. Scr. 6, 139.

Kerber, F., Nave, G., \& Sansonetti, C. J. 2008 ApJS 178, 374.

Kerber, F., Aldenius, M., Bristow, P, Nave, G., Ralchenko, Y., \& Sansonetti, C. J. this volume. Kurucz atomic line database http://www.cfa.harvard.edu/amp/ampdata/kurucz23/sekur.html

Litzén, U., Brault, J. W., \& Thorne, A. P. 1993, Phys. Scri. 47, 628

Nave, G., Johansson, S., Learner, R. C. M., Thorne, A. P, \& Brault, J. W. 1994, ApJS 94, 221

NIST Atomic Spectra Database. Ralchenko, Yu., Kramida, A. E., Reader, J. and NIST ASD Team 2008 [Online]. http://physics.nist.gov/asd3

O'Brian, T. R., Wickliffe, M. E., Lawler, J. E., Whaling, W., \& Brault, J. W. 1991 JOSA B 8, 1185.

Outred, M. 1978 J. Phys. Chem. Ref. data 7, 1.

Pickering, J. C., Thorne, A. P. T. 1996, ApJS 107, 761.

Pickering, J. C., Raassen, A. J. J., Uyling, P. H. M., Johansson, S. 1998, ApJS 117, 261.

Sansonetti, C. J., Blackwell, M. M., Saloman, E. B. 2004 J. Res. NIST 109, 371

Sansonetti, C. J. \& Green, M. B 2007, Phys. Scr. 75, 577.

VALD database: http://vald.astro.univie.ac.at/ vald/php/vald.php

Whaling W., Anderson W. H. C., Carle, M. T., Brault J. W., \& Zarem, H. A. 1995 J. Quant. Spectrosc. Radiat. transfer 53, 1

Whaling W., Anderson W. H. C., Carle, M. T., Brault J. W., \& Zarem, H. A. 2002, J. Res. NIST 107, 149. 